\title{
Observational properties of candidate supergiant fast X-ray transients
}

\author{
J.A. Zurita Heras* \\ Laboratoire AIM, CEA/DSM-CNRS-Université Paris Diderot, IRFU/Service d'Astrophysique, \\ FR-91191 Gif-sur-Yvette, France \\ E-mail: juan-antonio.zurita-herasecea.fr \\ S. Chaty \\ Laboratoire AIM, CEA/DSM-CNRS-Université Paris Diderot, IRFU/Service d'Astrophysique, \\ FR-91191 Gif-sur-Yvette, France \\ E-mail: sylvain. chaty@cea.fr

\section{R. Walter} \\ INTEGRAL Science Data Centre, Observatoire de Genève, Université de Genève, Chemin \\ d'Ecogia 16, 1290 Versoix, Switzerland \\ E-mail: roland.walter@unige.ch
}

INTEGRAL allowed the discovery of several new SuperGiant X-ray Binaries of which very few were known before its launch. Among them, INTEGRAL has unveiled a new sub-class: galactic $\mathrm{X}$-ray sources with a supergiant companion and displaying a transient behaviour, namely Supergiant Fast X-ray Transient. Most of the time in quiescence with a luminosity of $\lesssim 10^{33} \mathrm{erg} \mathrm{s}^{-1}$ (if not totally absent), these sources display rare episodes of short (few ks) and bright (up to $\sim 10^{36} \mathrm{erg} \mathrm{s}^{-1}$ as in other SGXB) flares. Several scenarii have been proposed to explain the atypical temporal behaviour of the SFXT among the SGXB: a different separation/geometry of their orbits, accretion of dense structures/clumps formed in the stellar wind that would produce these huge flares, magnetic centrifugal barrier and variation of the local stellar wind density and/or sources hosting a magnetar.

We present a systematic study, based on ISGRI observations from 2 ks-bin lightcurves to deep mosaics, of the observational properties (flares, duration, fluxes, etc.) of two SFXT candidates in order to bring constraints, to be able to distinguish between these different models of emission. Finally, this study will allow to better understand the overall characteristics of different classes of high-mass X-ray binaries.

7th INTEGRAL Workshop

September 8-11 2008

Copenhagen, Denmark

\footnotetext{
* Speaker.
} 


\section{Introduction}

Among the high-mass X-ray binaries (HMXBs), supergiant X-ray binaries (SGXBs) were divided in two classes depending on the accretion mechanism: 1) the Roche lobe overflow SGXBs with high luminosities of $\sim 10^{38} \mathrm{erg} \mathrm{s}^{-1}$, and 2) the wind-fed accreting SGXBs. These SGXBs are characterised by an $\mathrm{OB}$ supergiant companion, direct accretion onto the compact object from the strong stellar wind of the companion, a persistent luminosity of $\sim 10^{36} \mathrm{erg} \mathrm{s}^{-1}$, a high variability, and a circular orbital period of $\sim 3-15$ days. In most cases, the compact object is a neutron star with a strong magnetic field of $B \sim 10^{12-14} \mathrm{G}$ that modulates the $\mathrm{X}$-ray emission (reviews of $[1,2]$ and references therein).

Since its launch, INTEGRAL allowed the discovery of several new SGXBs (e.g. [3, 4]) with some of them showing an uncommon property: they were transient systems [5, 6]. They displayed rare and huge flares with peak luminosities of $\sim 10^{36} \mathrm{erg} \mathrm{s}^{-1}$. These flares showed sharp rises and decays of a few hundred seconds. Otherwise, they spent most of the time in a quiescent state with a luminosity of $\lesssim 10^{32-33} \mathrm{erg} \mathrm{s}^{-1}$, implying intensity dynamical range as large as $10^{3-4}$. Because of this behaviour, these likely-new sub-class of SGXBs were named supergiant fast X-ray transients (SFXTs, [7]).

Several mechanisms triggering such behaviour have been proposed. $[5,8,9]$ proposed that the compact object accretes from massive clumps formed in the stellar wind of the supergiant producing short and bright X-ray flares. Claiming that the Bondi-Hoyle accretion cannot reproduce the X-ray variability of IGR J11215-5952, [10] proposed an alternative model where the supergiant star would possess an anisotropic and denser equatorial wind in which the compact object accretes at each periastron passage similarly to the Be/X-ray binaries. [11] also predict that the sharp intensity transition observed during flares in SFXT are modulated by small variations of the stellar wind properties and the presence of a very strong magnetic field. Crossing the magnetic and/or centrifugal barriers would explain the fast transitions if the system hosts a slowly rotating pulsar with a magnetar-like field. Some clumpiness is also expected in this scenario.

We studied a sample of SFXT candidates in order to derive general observational properties. Herafter, we present our results on two sources: SAX J1818.6-1703 and AX J1845.0-0433. We describe the observations and data analysis in Sect. 2. We show the results and discuss them in Sect. 3. We conclude in Sect. 4.

\section{Observations and data analysis}

Our goal is to take advantage of the wide field of view $\left(29^{\circ} \times 29^{\circ}\right)$ of the hard X-ray and soft gamma-ray coded-mask spectro-imager ISGRI [12] on-board the INTEGRAL spacecraft [13] and of the long coverage of Galactic plane by the mission in order to study the SGXBs and SFXTs, and find the observational properties of these sources.

For this purpose, we make use of the ISGRI archive considering all the public data up to revolution 500 that means the analysis of $\sim 20000$ pointings. Individual sky images for each pointing were created in the $22-50 \mathrm{keV}$ energy range. We also built deep-exposure sky mosaics with exposures $\sim 10^{2-3} \mathrm{ks}$. As we treated each source separately at this step, the deep mosaics for each source were built disregarding all the pointings with a detection of the source. The count rate of each 
source was extracted in each image (pointings and mosaics) with the OSA tool mosaic_spec (version 1.7) where the position was fixed to the most accurate X-ray position and the point-spread function width was fixed to $6^{\prime}$. All detections of each source were considered above the $5 \sigma$ significance level. Detections and $5 \sigma$ upper limit were gathered to create long-term light curve of each source.

Moreover, a pointed observation with XMM-Newton was conducted on AX J1845.0-0433 on April 3, 2006, for a total exposure of $19 \mathrm{ks}$ and using the spectro-imager EPIC as main instrument. Event lists for each camera were built following standard procedures. Astrometry, timing and spectral analysis were performed on the data.

The $22-50 \mathrm{keV}$ count rate can be converted with the relation $1 \mathrm{Crab}=177.5$ counts $^{-1}=$ $9.2 \times 10^{-9} \mathrm{erg} \mathrm{cm}^{-2} \mathrm{~s}^{-1}$. The IJD time unit corresponds to MJD $=\mathrm{IJD}+51544$.

\section{Results}

The definition of the SFXT sub-class principally comes from the behaviour of the sources as observed by ISGRI. Discovered by INTEGRAL and identified as HMXB with a supergiant companion through multi-wavelength observations in X-rays, optical and infrared, these sources are rarely detected with ISGRI except during short and bright flares. Their quiescent emission reaching levels as low as $10^{32-33} \mathrm{erg} \mathrm{s}^{-1}[14,15]$, one needs observatories such as XMM-Newton or Swift to observe such low emission. We show 2 ISGRI light curves of one SGXB and one SFXT in Fig. 1 to illustrate the different temporal behaviour between both sub-classes.

\subsection{SAX J1818.6-1703}

The X-ray transient SAX J1818.6-1703 was discovered on March 11, 1998, during a bright flare observed with BeppoSAX [16]. INTEGRAL regularly observed the source field leading to several serendipitous detections of similar short (a few hours) and bright flares [17-21]. [5] reported its possible association with other fast X-ray transients like XTE J1739-302 and IGR J17544-2619, archetype of SFXTs. A particularly bright hard X-ray flare showed a complex structure with a short 10-20 min precursor peak and a main 1.5-2 $\mathrm{h}$ long peak with different intensities [18]. The hard $\mathrm{X}$-ray spectrum was also variable and became harder during the long flares [18]. With INTEGRAL/ISGRI and before March 2007, [8] listed 11 flares with duration of 2-6 ks and reported a quiescent level implying an intensity dynamic range of 250 between the highest flare and the quiescence. Using softer X-ray mission (Swift and Chandra), [22] and [23] reported a quiescent low level of $\sim(4-8) \times 10^{-12} \mathrm{erg} \mathrm{cm}^{-2} \mathrm{~s}^{-1}$, and an unabsorbed $0.5-10 \mathrm{keV}$ flux $3 \sigma$ upper limit of $1.1 \times 10^{-13} \mathrm{erg} \mathrm{cm}^{-2} \mathrm{~s}^{-1}$ with XMM-Newton implying an intensity dynamic range of $10^{3-4}$ as large as in other SFXTs. Searching for a reddened early-type star in optical and infrared catalogues, [24] proposed the source 2MASS J18183790-1702479 as the likely counterpart that was confirmed by Chandra [22]. The companion spectral type was constrained to a O9-B1 supergiant star [25].

The ISGRI 22-50 keV long-term light curve of SAX J1818.6-1703 is shown in Fig. 2. Detected in 32 individual pointings, the count rate was mostly between $(4.1-19.1) \pm 0.8 \mathrm{cts} \mathrm{s}^{-1}$. On Sept. 9, 2003, there were 2 particularly very bright flares that reached $44.8 \pm 0.8$ and $39.1 \pm$ $1.0 \mathrm{cts} \mathrm{s}^{-1}$ separated by $2 \mathrm{~h}$ (see [18]). Disregarding all these pointings, we built mosaics for each revolution where the source was only detected in 6 out of 191 revolutions with count rates between 


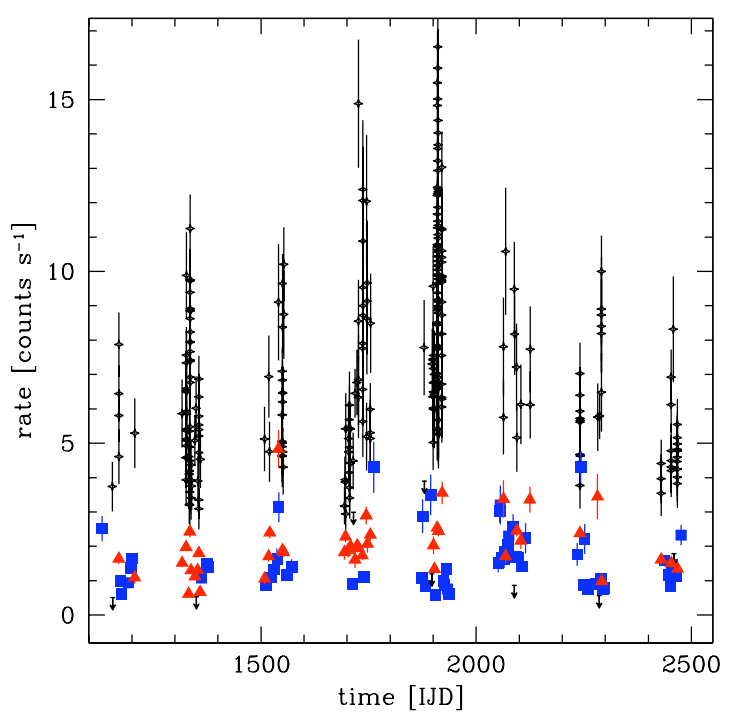

(a) IGR J17252-3616

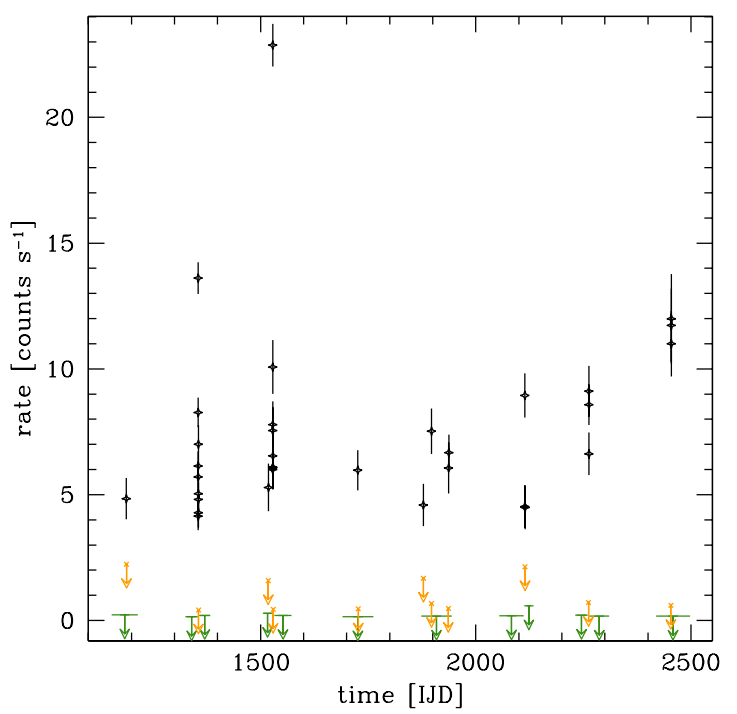

(b) IGR J17544-2619

Figure 1: Two examples of 22-50 keV long-term light curve of one classical persistent SGXB IGR J17252-3616 and one transient SGXB IGR J17544-2619, SFXT archetype. Detections in single pointings with exposure $\sim 2 \mathrm{ks}$ are shown with crosses and error bars. Disregarding the flares, the red triangles and blue boxes correspond to the average source count rate in deep mosaics with exposures of $10^{2-3} \mathrm{ks}$. Otherwise, $5 \sigma$ upper limits are represented (in yellow for exposure of $\sim 10^{2} \mathrm{ks}$, in green exposure of $\sim 10^{3} \mathrm{ks}$ ).

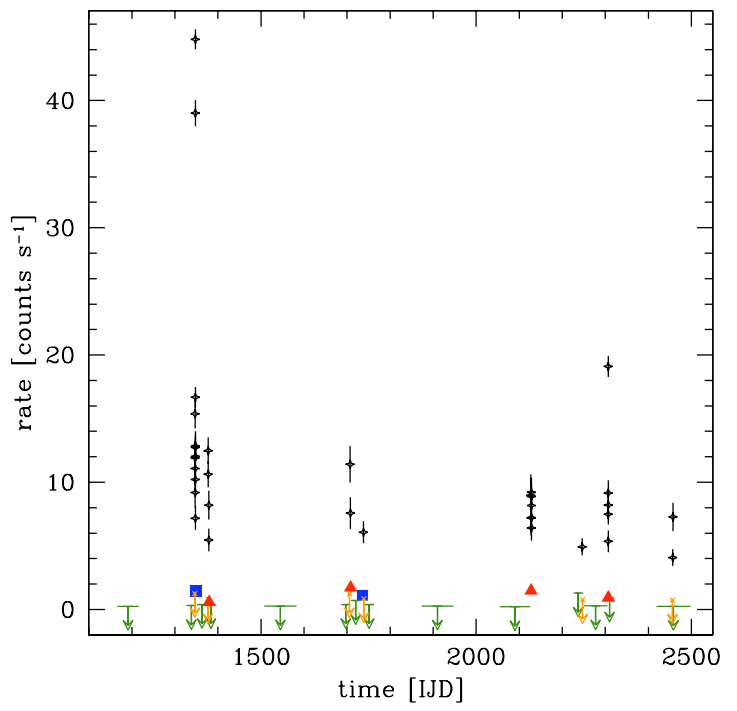

(a) $22-50 \mathrm{keV}$ long-term light curve

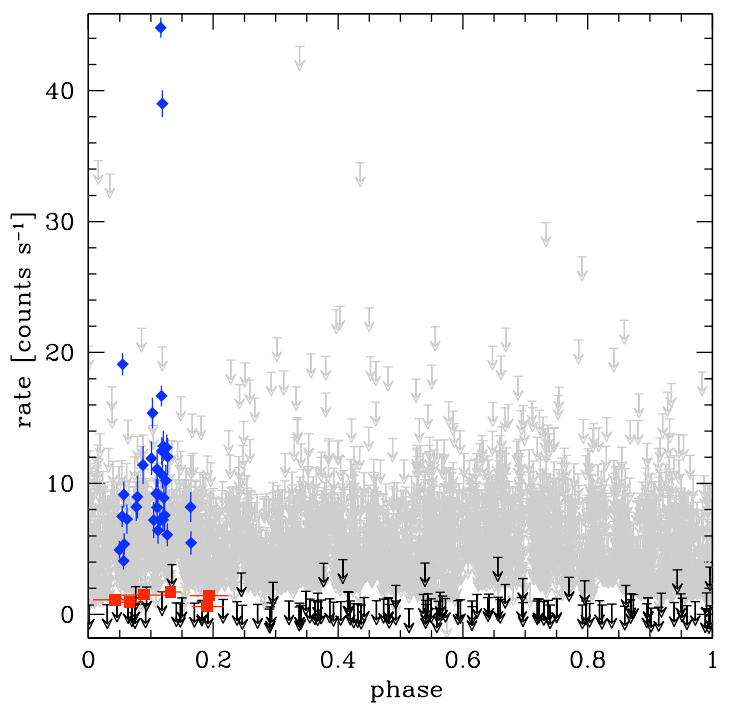

(b) $22-50 \mathrm{keV}$ folded light curve

Figure 2: left: ISGRI 22-50 keV light curve of SAX J1818.6-1703. See Fig. 1 for the points explanation. right: Folded light curve with T0=IJD 1133.7365. Crosses are detections in single pointings and the light $5 \sigma$ upper limits are non-detections in all the other pointings. Boxes are detections in revolution deep mosaics, and dark $5 \sigma$ upper limits are non-detections in the other revolution deep mosaics. 
$(0.9 \pm 0.1)-(1.7 \pm 0.3) \mathrm{cts} \mathrm{s}^{-1}$ (triangles and boxes in Fig. 2). We searched for other detections accumulating consecutive revolutions in very-long exposure mosaics. However, no other detection was observed with an average $5 \sigma$ upper limit of $0.3 \mathrm{cts} \mathrm{s}^{-1}$ (large $5 \sigma$ upper limits in Fig. 2). The source was detected in 8 different epochs for a few days at most. A separation of $\sim 30$ days is observed between detections 1-2 and 3-4, and of $\sim 60 \mathrm{~d}$ between 6-7.

The Fourier analysis (Lomb-Scargle periodogram, fast method of [26]) revealed a period of $30.0 \pm 0.2$ d. The folded light curve shown in Fig. 2 showed that all the detections were comprised between phases $0.0-0.2$, so an elapsed time of 6 days. Of 31 revolutions falling in this phase interval, there was at least one detection of the source in 12 of them. The combination of the other 19 revolutions in one deep mosaic revealed the source with its lowest hard X-ray intensity of $0.47 \pm 0.06 \mathrm{cts} \mathrm{s}^{-1}$. Combining revolutions per step of 0.2 phase between $0.2-1.0$ (4 mosaics with exposure 1.5-2 Ms each), the source was not detected with a $5 \sigma$ upper limit of $\sim 0.2 \mathrm{cts} \mathrm{s}^{-1}$. The detections from the softer X-ray missions $[22,23]$ also occurred within the phase interval 0.00.2. On the other hand, the XMM-Newton upper limit [23] occurred at the apastron passage at phase 0.58 . Finally, a modulation of activity at a consistent period is found (D.M. Smith, private communication) in the RXTE Galactic bulge scan data [27].

As SAX J1818.6-1703 is a confirmed SGXB, one can interpret that the compact object follows an eccentric orbit around the supergiant star with a $30 \mathrm{~d}$ orbital period, and with a short accretion episode of $6 \mathrm{~d}$ occurring at each periastron passage. Only during the accretion phase do the source behave as the other classical SGXBs with a persistent emission and an important variability of factor 10-50. Only for very few flares did the source vary by a factor $>100$ as observed in SFXTs.

\subsection{AX J1845.0-0433}

AX J1845.0-0433 was discovered as a transient and highly-variable X-ray source with ASCA [28]. Because of its absorbed and flat X-ray spectrum during the flare, the source was assimilated to accreting pulsars, even if no coherent pulsation was observed for periods between $125 \mathrm{~ms}$ and $4096 \mathrm{~s}$ [28]. [29] pinpointed the optical counterpart as being a O9.5I supergiant star located $\sim$ $3.6 \mathrm{kpc}$ away. Being a transient SGXB, [7] considered it an SFXT candidate. New flaring activity with INTEGRAL [30, 31] and Swift [32] was reported. The flare duration was typically of a few tens of minutes with sharp-rising/decaying times of a few minutes and the X-ray spectra was represented with similar absorbed flat power laws confirming the SFXT classification [32].

The $22-50 \mathrm{keV}$ long-term variability of AX J1845.0-0433, built with the same procedure as for SAX J1818.6-1703, is shown in Fig. 3). The source was only detected in 8 pointings out of 2597 with count rates within $3-6 \mathrm{cts} \mathrm{s}^{-1}$ and randomly distributed along the $3.5 \mathrm{yr}$ observations. From the deep mosaics per revolution (exposure $\sim 100 \mathrm{ks}$ ), there were only two detections with $0.6 \pm 0.1 \mathrm{cts} \mathrm{s}^{-1}$, coincident in time with flares and implying intensity dynamic range of factor 510. Cumulating data over several consecutive revolutions (mosaic exposures of $\sim 1 \mathrm{Ms}$ ) revealed two other detections with $0.20 \pm 0.04 \mathrm{cts} \mathrm{s}^{-1}$ and $0.33 \pm 0.06 \mathrm{cts} \mathrm{s}^{-1}$, respectively. Otherwise, cumulating all the data without detections, the source was detected with $0.16 \pm 0.02 \mathrm{cts} \mathrm{s}^{-1}$, implying a maximum intensity dynamic range of 40 between the brightest flare and the quiescent level. The bright flare frequency can be estimated as one every $4-5$ days. 


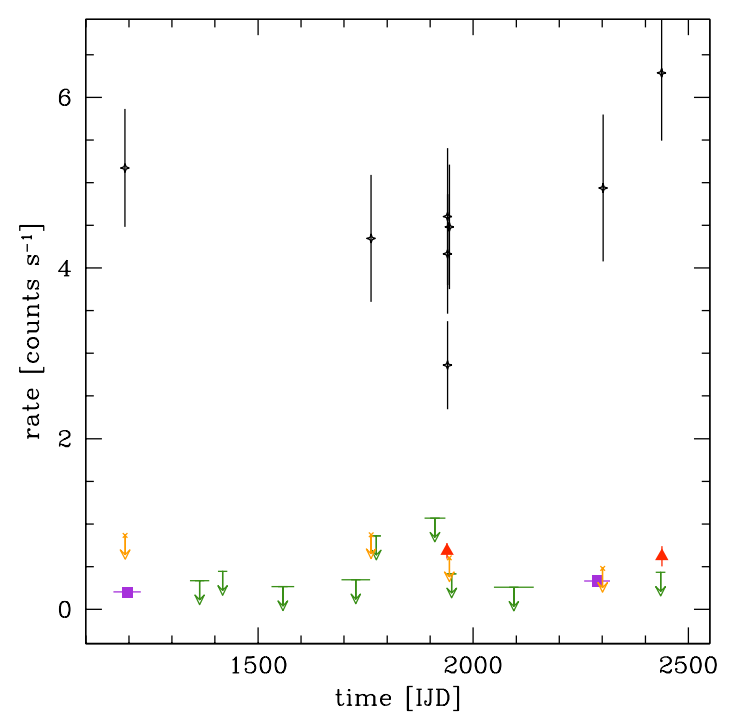

(a) ISGRI 22-50 keV long-term light curve

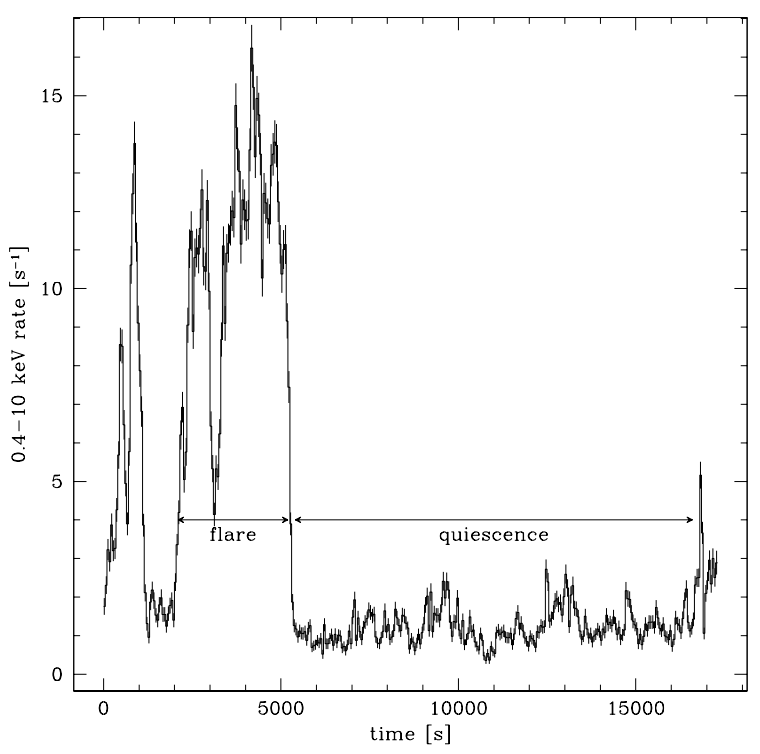

(b) EPIC/pn 50 s binned light curve

Figure 3: left: ISGRI 22-50 keV light curve of AX J1845.0-0433. See Fig. 1 for the points explanation. Violet boxes are detections in mosaics with exposure $\sim 1 \mathrm{Ms}$. right: EPIC/pn light curve with $\mathrm{T} 0=$ MJD 53828.64299 and bin $=50 \mathrm{~s}$.

The EPIC/pn 0.4-10 keV light curve shows two different states of the source in Fig. 3. Two short and complex flares of durations $1 \mathrm{ks}$ and $3 \mathrm{ks}$, respectively, happened during the first $5 \mathrm{ks}$ observation. In both flare, the intensity sharply rose and decayed between $\sim 2-12 \mathrm{cts} \mathrm{s}^{-1}$ on time scales of few hundred seconds with also short peaks reaching $\sim 15 \mathrm{cts} \mathrm{s}^{-1}$ (maximum $16.2 \pm$ $\left.0.6 \mathrm{cts} \mathrm{s}^{-1}\right)$. Moreover, abrupt and short $\left(\lesssim 300 \mathrm{~s}\right.$ ) decays of the intensity down to $\sim 4 \mathrm{cts} \mathrm{s}^{-1}$ happened within the flares but without reaching the quiescent level. The flare durations are comparable to those observed by INTEGRAL. Then, the source reached its average quiescent level of $1.2 \pm 0.2 \mathrm{cts} \mathrm{s}^{-1}$, showing continuous variability of a factor 2-3 with several peaks $>2 \mathrm{cts} \mathrm{s}^{-1}$. The variability factor between the lowest and highest $0.4-10 \mathrm{keV}$ count rate was 45 . No coherent pulsation in the range $0.1-5000 \mathrm{~s}$ was observed, neither during the flare nor during the quiescence phase.

\subsection{Accretion onto transient SGXBs: the cases of SAX J1818.6-1703 and AX J1845.0-0433}

Assuming a smooth wind ${ }^{1}$, the Bondi-Hoyle accretion ${ }^{2}$ (see Fig. 2 for the case B0.5I), and an elliptical orbit ${ }^{3}$, one can extrapolate about the geometry of SAX J1818.6-1703 and pinpoint the likely spectral-type of the companion. Considering typical stellar fundamental parameters of O9I-B1I stars [34, 35], the expected accretion rates are similar with a variation factor less than 3

\footnotetext{
${ }^{1}$ approximated as $v_{\text {wind }}(r)=v_{\infty}\left(1-R_{*} / r\right)^{\beta}$ where $v_{\infty}$ is the terminal velocity, $R_{*}$ the stellar radius, and $\beta=1$.

${ }^{2}$ luminosity approximated to $L_{\mathrm{X}}=\varepsilon \dot{M}_{\text {accr }} c^{2}$ with the accretion efficiency $\varepsilon \sim 0.2$, the accretion rate $\dot{M}_{\text {accr }}=$ $\left(R_{\text {accr }}^{2} / 4 a^{2}\right) \dot{M}_{\text {wind }}$, the stellar mass-loss rate $\dot{M}_{\text {wind }}$ from eq. 12 of [33], the accretion radius $R_{\text {accr }}=2 G M_{\mathrm{X}} /\left(v_{\text {wind }}^{2}+v_{\mathrm{X}}^{2}\right)$, and $v_{\mathrm{X}}$ is the compact object velocity.

${ }^{3}$ semi-major axis $a$ of the system determined with $a^{3} / P^{2}=G\left(M_{*}+M_{\mathrm{X}}\right) / 4 \pi^{2}$ with the period $P$, the stellar mass $M_{*}$, and the mass of the compact object fixed to $M_{\mathrm{X}}=1.4 M_{\odot}$.
} 


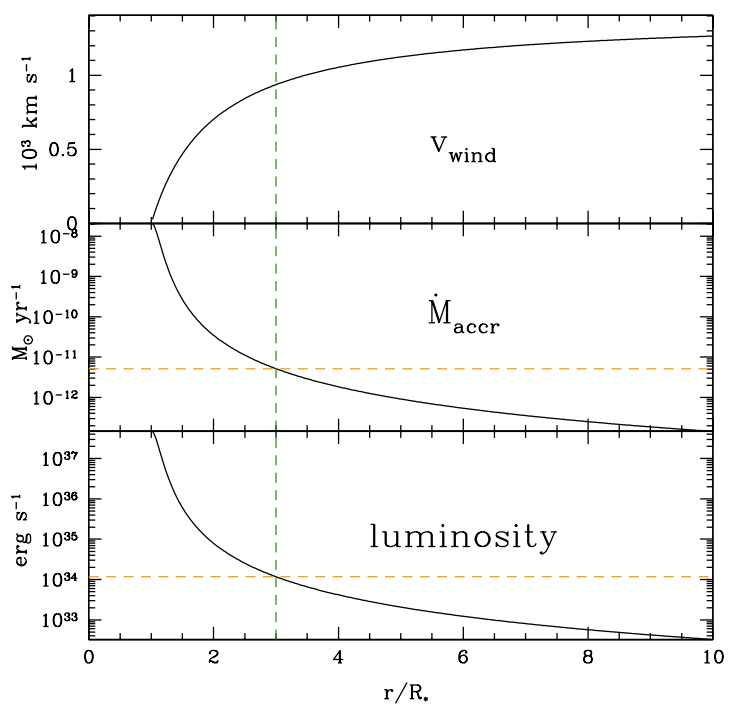

(a) B0.5I star
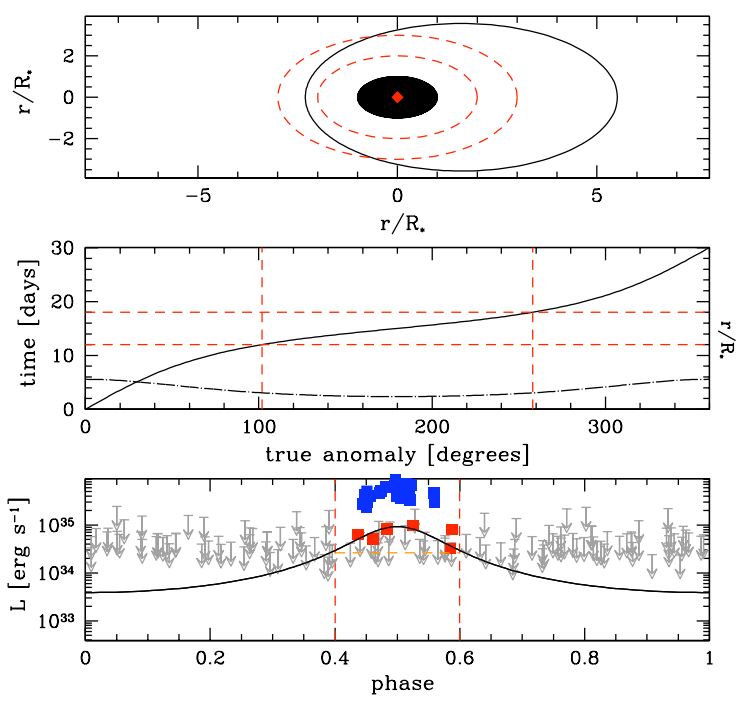

(b) Eccentric orbit

Figure 4: Case for SAX J1818.6-1703. left: Smooth wind+Bondi-Hoyle accretion models where the wind velocity $\left(v_{\text {wind }}\right)$, the accreted mass rate $\left(\dot{M}_{\text {accr }}\right)$, and the X-ray luminosity are shown. right: At top, elliptical orbit with eccentricity $e=0.41$ shown as a solid line, and dashed lines are 2 and $3 R_{*}$ circles. In the middle, orbital time vs phase is shown in solid line, and binary separation $r / R_{*}$ vs phase is shown in long-dashed line. The dashed lines represent the accretion zone with $r \leq 3 R_{*}$ (vertical) and the $6 \mathrm{~d}$ accretion time (horizontal). At the bottom, the folded light curve shown in Fig. 2 is over-plotted on the expected Bondi-Hoyle luminosity (solid line). The vertical dashed lines represent $t_{\text {accr }}$ and the horizontal one marks the lowest $22-50 \mathrm{keV}$ count rate of the source detected during the periastron passage.

between spectral types. The key parameter is the distance between the star and the compact object where the accretion rate can vary by a factor up to $\sim 10$ between $r=2-3 R_{*}$. Considering the flux from the X-ray missions [22, 23], an accretion zone of size $r \leq 3 R_{*}$ is consistent with the observations if the system is located $<8 \mathrm{kpc}$. However, the size of the accretion zone may vary up to $1 R_{*}$ as the stellar mass-loss rate can change by a factor of 2-3 in supergiants with identical spectral type [35].

An elliptical orbit of $P=30.0 \pm 0.2 \mathrm{~d}$ and an elapsed time of accretion $t_{\mathrm{accr}}=6 \mathrm{~d}$ must constrain the eccentricity $e$ of the binary system depending on the stellar parameters such as the mass and the radius of the companion star. For O9I, O9.5I and B0Ia, the maximum elapsed accretion times allowed by the stellar parameters are always shorter than the $t_{\text {accr }}$ derived above for any eccentricity. For a B0.5Ia star, the conditions are fulfilled with $e=0.41$ or $e=0.58$ that imply a perihelionaphelion of 2.3-5.5 $R_{*}$ or 1.6-6.2 $R_{*}$, respectively (see Fig. 4). For a B1Ia star, the conditions are fulfilled with $e=0.25$ (i.e. $r \in 2.7-4.5 R_{*}$ ) or $e=0.73$ (i.e. $r \in 1.0-6.2 R_{*}$ ). Disregarding the short and bright flares, the hard X-ray flux varies between $\sim(2-8) \times 10^{-11} \mathrm{erg} \mathrm{cm}^{-2} \mathrm{~s}^{-1}$ along the whole $t_{\text {accr }}$. Therefore, the compact object-companion star distance $r$ should not vary by a high factor when crossing the accretion zone, thus favouring low eccentricities (see Fig. 4). Therefore, the case of a B0.5-1Ia companion star with a low eccentricity ( $e=0.41$ or 0.25 , respectively) are the best solutions to reconcile the wind model and observations. This determination is accurate to 
0.5 spectral type since a wider accretion zone of $4 R_{*}$ would also give a solution for a B0I star with $e=0.16$.

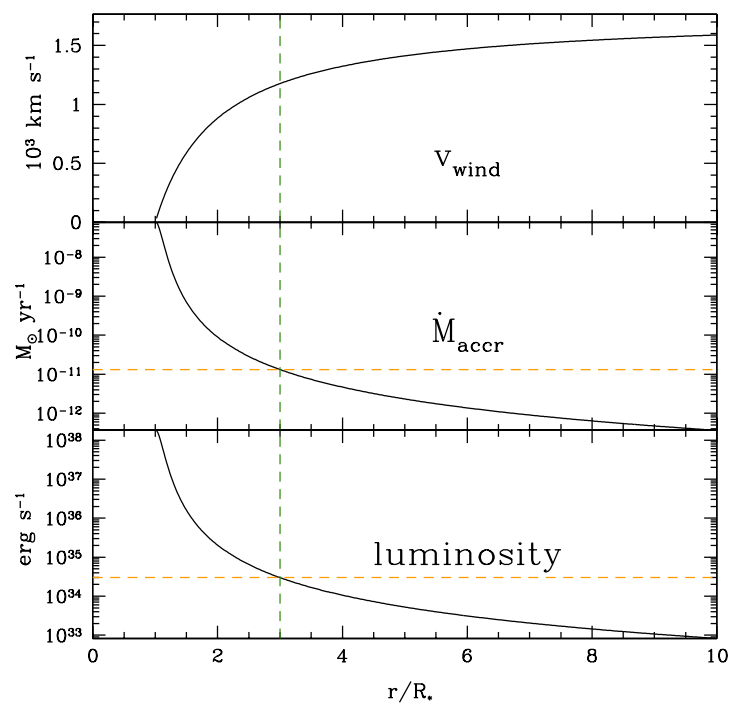

(a) O9.5I star
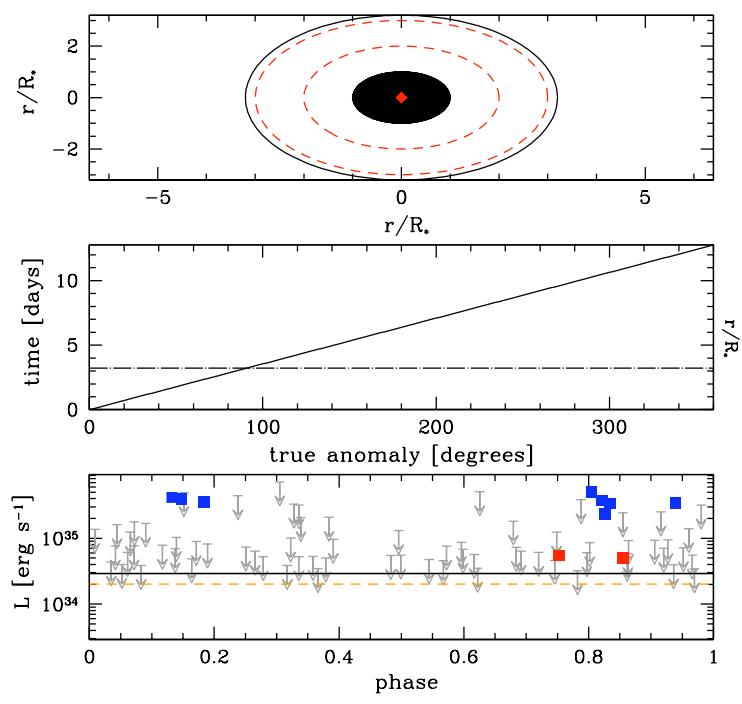

(b) Circular orbit

Figure 5: Case for AX J1845.0-0433. See also Fig. 4 for points/lines explanation. left: Smooth wind+Bondi-Hoyle accretion models. right: At top, circular orbit with radius $3.2 R_{*}$ derived from the observed luminosity and Bondi-Hoyle luminosity. At the bottom, the folded light curve (assuming a circular orbit of $3.2 R_{*}$ radius and system seen face-on) is over-plotted on the expected Bondi-Hoyle luminosity (solid line). The dashed line marks the lowest $22-50 \mathrm{keV}$ count rate of the source.

Assuming a distance of $3.6 \mathrm{kpc}$, the quiescent average $0.4-10$ and $20-100 \mathrm{keV}$ luminosities of AX J1845.0-0433 are $1.4 \times 10^{35} \mathrm{erg} \mathrm{s}^{-1}$ and $2.7 \times 10^{34} \mathrm{erg} \mathrm{s}^{-1}$, respectively. This EPIC quiescent luminosity is compatible with the ones observed with Swift and ASCA. During the flares, the average 0.4-10 and 20-50 keV luminosities are $1.8 \times 10^{36} \mathrm{erg} \mathrm{s}^{-1}$ and $4.5 \times 10^{35} \mathrm{erg} \mathrm{s}^{-1}$, respectively. Since these luminosities are too high to be explained by the X-ray luminosity of $10^{30-33} \mathrm{erg} \mathrm{s}^{-1} \mathrm{de}-$ tected in single OB stars [36], the source must be permanently accreting matter as the high-energy emission is persistent. With such persistent quiescent X-ray luminosity of $\lesssim 2 \times 10^{35} \mathrm{erg} \mathrm{s}^{-1}$ in AX J1845.0-0433, one needs a binary separation of $a \gtrsim 5 \times 10^{12} \mathrm{~cm} \sim 3 R_{*}$, that is higher than in classical SGXBs $\left(a \approx 2 R_{*}\right)$ when assuming the smooth wind model, the Bondi-Hoyle accretion, and a circular orbit (models shown in Fig. 5).

\subsection{Massive clumps}

For both sources, several high-energy missions observed short and bright flares implying intensity dynamic range $\sim 50$ times higher than the average level during the accretion phase. $[5,8,9]$ showed that such huge flares in transient SGXBs resulted from the accretion of massive clumps formed within the stellar wind. Indeed, instabilities of the radiation line-driven outflows of hot stars generate structures within the stellar wind affecting parameters such as the wind velocity, the outflow density, or the mass-loss rate [37]. Recently, [38] have shown that optically-thick macro-clumps must form within the wind of hot supergiant stars as it affects the strength of some 
$\mathrm{X}$-ray/UV lines emission of a supergiant star. Determining average parameters of luminosity, frequency, and duration of flares in SFXTs from INTEGRAL observations, [8] derived clump parameters that agreed with this macro-clump scenario developed by [38], and predicted that accretion in SGXBs should follow a two-regime behaviour depending on whether the distance between the compact object and the supergiant companion was bigger or smaller than $\sim 2-3 R_{*}$ (see also [9]). [9] showed that the number of clumps that a compact object could statistically accrete within one orbit around a O8-B0I star varies between 1-8 with an orbital radius $a=3-6 R_{*}$. These values agree with the flare frequency observed in SAX J1818.6-1703 and AX J1845.0-0433. In the case of SAX J1818.6-1703, it can also explain the observation of flares slightly outside the accretion phase as the flare observed by BeppoSAX.

\section{Conclusion}

Timing analysis with INTEGRAL revealed an orbital period of $30.0 \pm 0.2 \mathrm{~d}$ and an elapsed accretion phase of $\sim 6 \mathrm{~d}$ in the transient SGXB SAX J1818.6-1703. That implies an elliptical orbit and constrains the possible supergiant spectral type between B0-0.5I with eccentricities $e \sim$ $0.3-0.6$ (for average fundamental parameters of supergiant stars). During the accretion phase, the behaviour of the source is in agreement with other SGXB. The huge variations of the observed $\mathrm{X}$-ray intensity can be explained through the accretion of macro-clumps formed within the stellar wind.

AX J1845.0-0433 is a persistent X-ray binary with a O9.5I supergiant companion emitting at a rather low $0.2-100 \mathrm{keV}$ luminosity of $\sim 10^{35} \mathrm{erg} \mathrm{s}^{-1}$ with seldom flares reaching luminosities of $\sim 10^{36} \mathrm{erg} \mathrm{s}^{-1}$. Variability factors as high as 50 are observed on very short-time scale (few ks). The flare characteristics (number, luminosity, duration, increase/decrease time) in contrast to the persistent quiescent emission suggest that clumps are formed within the stellar wind of the supergiant companion.

SAX J1818.6-1703 and AX J1845.0-0433 reinforce the model that predicts that SFXT are SGXB moving in orbits differing from previously known SGXB systems (either a higher circular orbital radius or an eccentric orbit). For SAX J1818.6-1703, further observations either in Xrays or optical/infrared wavelengths are needed in order to confirm our estimation and to constrain the parameters of the system. That will help to elucidate what are the mechanisms triggering the transient behaviour.

\section{References}

[1] N. E. White, F. Nagase, and A. N. Parmar, X-ray binaries, pp. 1-57. Cambridge Astrophysics Series, Cambridge, MA: Cambridge University Press, Ic1995, edited by Lewin, Walter H.G.; Van Paradijs, Jan; Van den Heuvel, Edward P.J., 1995.

[2] D. Psaltis, Accreting neutron stars and black holes: a decade of discoveries, pp. 1-38. Compact stellar X-ray sources, Apr., 2006.

[3] R. Walter, J. Zurita Heras, L. Bassani, A. Bazzano, A. Bodaghee, A. Dean, P. Dubath, A. N. Parmar, M. Renaud, and P. Ubertini, XMM-Newton and INTEGRAL observations of new absorbed supergiant high-mass X-ray binaries, A\&A 453 (July, 2006) 133-143. 
[4] S. Chaty, F. Rahoui, C. Foellmi, J. A. Tomsick, J. Rodriguez, and R. Walter, Multi-wavelength observations of Galactic hard X-ray sources discovered by INTEGRAL. I. The nature of the companion star, A\&A 484 (June, 2008) 783-800, [0802 .1774].

[5] J. J. M. in't Zand, Chandra observation of the fast X-ray transient IGR J17544-2619: evidence for a neutron star?, A\&A 441 (Oct., 2005) L1-L4, [arXiv: astro-ph/ 0508240 ].

[6] D. M. Smith, W. A. Heindl, C. B. Markwardt, J. H. Swank, I. Negueruela, T. E. Harrison, and L. Huss, XTE J1739-302 as a Supergiant Fast X-Ray Transient, ApJ 638 (Feb., 2006) 974-981, [astro-ph/0510658].

[7] I. Negueruela, D. M. Smith, P. Reig, S. Chaty, and J. M. Torrejón, Supergiant Fast X-ray Transients: a new class of high mass X-ray binaries unveiled by INTEGRAL, in ESA SP-604: The X-ray Universe 2005 (A. Wilson, ed.), pp. 165-170, Jan., 2006.

[8] R. Walter and J. Zurita Heras, Probing clumpy stellar winds with a neutron star, A\&A 476 (Dec., 2007) 335-340, [arXiv:0710.2542].

[9] I. Negueruela, J. M. Torrejón, P. Reig, M. Ribó, and D. M. Smith, Supergiant Fast X-ray Transients and Other Wind Accretors, in American Institute of Physics Conference Series, vol. 1010 of American Institute of Physics Conference Series, pp. 252-256, May, 2008.

[10] L. Sidoli, P. Romano, S. Mereghetti, A. Paizis, S. Vercellone, V. Mangano, and D. Götz, An alternative hypothesis for the outburst mechanism in supergiant fast X-ray transients: the case of IGR J11215-5952, A\&A 476 (Dec., 2007) 1307-1315, [arXiv: 0710.1175$].$

[11] E. Bozzo, M. Falanga, and L. Stella, Are There Magnetars in High-Mass X-Ray Binaries? The Case of Supergiant Fast X-Ray Transients, ApJ 683 (Aug., 2008) 1031-1044, [0 805.1849 ].

[12] F. Lebrun, J. P. Leray, P. Lavocat, J. Crétolle, M. Arquès, C. Blondel, C. Bonnin, A. Bouère, C. Cara, T. Chaleil, F. Daly, F. Desages, H. Dzitko, B. Horeau, P. Laurent, O. Limousin, F. Mathy, V. Mauguen, F. Meignier, F. Molinié, E. Poindron, M. Rouger, A. Sauvageon, and T. Tourrette, ISGRI: The INTEGRAL Soft Gamma-Ray Imager, A\&A 411 (Nov., 2003) L141-L148.

[13] C. Winkler, T. J.-L. Courvoisier, G. Di Cocco, N. Gehrels, A. Giménez, S. Grebenev, W. Hermsen, J. M. Mas-Hesse, F. Lebrun, N. Lund, G. G. C. Palumbo, J. Paul, J.-P. Roques, H. Schnopper, V. Schönfelder, R. Sunyaev, B. Teegarden, P. Ubertini, G. Vedrenne, and A. J. Dean, The INTEGRAL mission, A\&A 411 (Nov., 2003) L1-L6.

[14] D. Götz, M. Falanga, F. Senziani, A. De Luca, S. Schanne, and A. von Kienlin, IGR J08408-4503: A New Recurrent Supergiant Fast X-Ray Transient, ApJ 655 (Feb., 2007) L101-L104, [arXiv:astro-ph/0612437].

[15] J.-C. Leyder, R. Walter, M. Lazos, N. Masetti, and N. Produit, Hard X-ray flares in IGR J08408-4503 unveil clumpy stellar winds, A\&A 465 (Apr., 2007) L35-L38.

[16] J. in 't Zand, J. Heise, M. Smith, J. M. Muller, P. Ubertini, and A. Bazzano, SAX J1818.6-1703 and KS 1741-293, IAU Circ. 6840 (Mar., 1998) 2-+.

[17] M. G. Revnivtsev, R. A. Sunyaev, D. A. Varshalovich, V. V. Zheleznyakov, A. M. Cherepashchuk, A. A. Lutovinov, E. M. Churazov, S. A. Grebenev, and M. R. Gilfanov, A Hard X-ray Survey of the Galactic-Center Region with the IBIS Telescope of the INTEGRAL Observatory: A Catalog of Sources, Astronomy Letters 30 (June, 2004) 382-389, [arXiv: astro-ph / 0402027 ].

[18] S. A. Grebenev and R. A. Sunyaev, Outburst of the X-ray Transient SAX J1818.6-1703 Detected by the INTEGRAL Observatory in September 2003, Astronomy Letters 31 (Oct., 2005) 672-680, [arXiv:astro-ph/0509133]. 
[19] V. Sguera, E. J. Barlow, A. J. Bird, D. J. Clark, A. J. Dean, A. B. Hill, L. Moran, S. E. Shaw, D. R. Willis, A. Bazzano, P. Ubertini, and A. Malizia, INTEGRAL observations of recurrent fast X-ray transient sources, A\&A 444 (Dec., 2005) 221-231, [arXiv: astro-ph/ 0509018 ].

[20] E. Kuulkers, S. E. Shaw, A. Paizis, J. Chenevez, S. Brandt, T. J.-L. Courvoisier, A. Domingo, K. Ebisawa, P. Kretschmar, C. B. Markwardt, N. Mowlavi, T. Oosterbroek, A. Orr, D. Rísquez, C. Sanchez-Fernandez, and R. Wijnands, The INTEGRAL Galactic bulge monitoring program: the first 1.5 years, A\&A 466 (May, 2007) 595-618, [arXiv: astro-ph/ 0701244 ].

[21] S. A. Grebenev and R. A. Sunyaev, Increasing X-ray activity of SAX J1818.6-1703 detected by INTEGRAL, The Astronomer's Telegram 1482 (Apr., 2008) 1-+.

[22] J. in't Zand, P. Jonker, M. Mendez, and C. Markwardt, Chandra observation of SAX J1818.6-1703: confirmation of optical counterpart \& suggestion of non-transient nature, The Astronomer's Telegram 915 (Oct., 2006) 1-+.

[23] E. Bozzo, S. Campana, L. Stella, M. Falanga, G. Israel, R. Rampy, D. Smith, and I. Negueruela, XMM-Newton \& SWIFT observations of the Supergiant Fast X-ray transient SAXJ1818.6-1703, The Astronomer's Telegram 1493 (Apr., 2008) 1-+.

[24] I. Negueruela and D. M. Smith, Optical counterpart to SAX J1818.6-1703, The Astronomer's Telegram 831 (June, 2006) 1-+.

[25] I. Negueruela and M. P. E. Schurch, A search for counterparts to massive X-ray binaries using photometric catalogues, A\&A 461 (Jan., 2007) 631-639, [arXiv: astro-ph/ 0610006 ].

[26] W. H. Press and G. B. Rybicki, Fast algorithm for spectral analysis of unevenly sampled data, ApJ 338 (Mar., 1989) 277-280.

[27] C. B. Markwardt, J. H. Swank, F. E. Marshall, and J. J. M. in't Zand, RXTE Monitoring and "Imaging” Observations of the Galactic Bulge, in Rossi2000: Astrophysics with the Rossi X-ray Timing Explorer (T. E. Strohmayer, ed.), 2000.

[28] S. Yamauchi, T. Aoki, K. Hayashida, H. Kaneda, K. Koyama, M. Sugizaki, Y. Tanaka, H. Tomida, and Y. Tsuboi, New Transient X-Ray Source in the Scutum Region Discovered with ASCA, PASJ 47 (Apr., 1995) 189-194.

[29] M. J. Coe, J. Fabregat, I. Negueruela, P. Roche, and I. A. Steele, Discovery of the optical counterpart to the ASCA transient AX 1845.0-0433, MNRAS 281 (July, 1996) 333-338.

[30] S. V. Molkov, A. M. Cherepashchuk, A. A. Lutovinov, M. G. Revnivtsev, K. A. Postnov, and R. A. Sunyaev, A Hard X-ray Survey of the Sagittarius Arm Tangent with the IBIS Telescope of the INTEGRAL Observatory: A Catalog of Sources, Astronomy Letters 30 (Aug., 2004) 534-539, [astro-ph/0402416].

[31] J. P. Halpern and E. V. Gotthelf, Igr J18450-0435 = AX J18450-0433, The Astronomer's Telegram 692 (Jan., 2006) 1-+.

[32] V. Sguera, A. J. Bird, A. J. Dean, A. Bazzano, P. Ubertini, R. Landi, A. Malizia, E. J. Barlow, D. J. Clark, A. B. Hill, and M. Molina, INTEGRAL and Swift observations of the supergiant fast X-ray transient AX J1845.0-0433 = IGR J18450-0435, A\&A 462 (Feb., 2007) 695-698, [astro-ph/0610880].

[33] J. S. Vink, A. de Koter, and H. J. G. L. M. Lamers, New theoretical mass-loss rates of $O$ and B stars, A\&A 362 (Oct., 2000) 295-309, [arXiv: astro-ph/ 0008183 ]. 
[34] F. Martins, D. Schaerer, and D. J. Hillier, A new calibration of stellar parameters of Galactic O stars, A\&A 436 (June, 2005) 1049-1065, [arXiv: astro-ph/ 0503346 ].

[35] P. A. Crowther, D. J. Lennon, and N. R. Walborn, Physical parameters and wind properties of galactic early B supergiants, A\&A 446 (Jan., 2006) 279-293, [arXiv : astro-ph/ 0509436 ].

[36] T. W. Berghoefer, J. H. M. M. Schmitt, R. Danner, and J. P. Cassinelli, X-ray properties of bright OB-type stars detected in the ROSAT all-sky survey., A\&A 322 (June, 1997) 167-174.

[37] M. C. Runacres and S. P. Owocki, The outer evolution of instability-generated structure in radiatively driven stellar winds, A\&A 381 (Jan., 2002) 1015-1025.

[38] L. M. Oskinova, W.-R. Hamann, and A. Feldmeier, Neglecting the porosity of hot-star winds can lead to underestimating mass-loss rates, A\&A 476 (Dec., 2007) 1331-1340, [arXiv: 0704 . 2390]. 